\title{
Dose estimation in reference and non-reference pediatric patients undergoing computed tomography examinations: a Monte Carlo study
}

\author{
P. Akhlaghi, H. Miri-Hakimabad and L. Rafat-Motavalli ${ }^{\star}$ \\ Physics Department, Faculty of Sciences, Ferdowsi University of Mashhad, 91775-1436 Mashhad, Iran.
}

Received 16 April 2014 - Accepted 26 August 2014

\begin{abstract}
The global increase in the number of computed tomography (CT) examinations have enhanced concerns regarding stochastic radiation risks to patients, especially for children. Considering that cancer risk is cumulative over a lifetime and each CT examination contributes to the lifetime exposure, there is a need for a better understanding of radiation-induced cancer incidence and mortality, and better dose estimates. Accordingly, some authors estimated organ and effective dose in reference phantoms, but still there is a critical need to expand these data to larger groups of non-reference children. As an initial step to address this issue, in this study organ and effective doses were calculated in common CT procedures in non-reference pediatric phantoms and were compared with those of reference phantoms with the similar ages. Thirteen pediatric phantoms, BABY CHILD, five voxel-based UF pediatric phantoms (B-series) and six phantoms developed at The Foundation for Research on Information Technologies in Society (IT'IS) were implemented into MCNP. According to the results, there were no consistent differences between the doses of organs exposed indirectly and effective doses of these three phantom types, but it was observed that for organs located in the scan region, there was a relation between absorbed doses and pediatric age group, as expected. Generally, using the results of this study one can estimate the absorbed doses more accurately. But it should be noted that these low expansion data are not comprehensive enough for finding a reasonable relationship between phantom size and effective dose except in chest-abdomen-pelvis (CAP) imaging.
\end{abstract}

Keywords: Pediatric computed tomography / reference and non-reference phantoms / age and size dependent absorbed dose / effective dose estimation

\section{Introduction}

Computed tomography (CT) examinations at very high resolution completed within a few seconds are routinely available today and offer excellent diagnostic capabilities. Not surprisingly, these developments led to a strong increase in the clinical use of CT (Kalender, 2014). The increased use of CT examinations has raised concerns, regarding enhanced radiation dose and associated stochastic cancer risk to patients (Brenner and Hall, 2007; Berrington de Gonzàlez et al., 2009) especially for children (Brenner, 2002; ICRP, 2007a).

In response to this concern, the U.S. Food and Drug Administration (FDA) announced the "Initiative to Reduce Radiation Exposure from Medical Imaging", which focuses on increasing the safety and making informed decision about the use of CT examinations (USFDA, 2010). According to Brenner and Hall article, the cancer risk is cumulative over a lifetime. Each CT examination (including multiple series per examination) contributes to the lifetime exposure. Therefore,

\footnotetext{
* rafat@ferdowsi.um.ac.ir
}

there is a need for a better understanding of radiation-induced cancer incidence and mortality, and better dose estimates (Brenner and Hall, 2007).

Although, estimating the organ doses with high accuracy are demanding, but they are not necessary for routine purposes (Kalender, 2014). Such efforts in developing database for radiation dose would be used to identify patients whose cumulative lifetime dose has reached higher levels from frequent imaging studies involving exposure to ionizing radiation. The information may be used to determine when alternative imaging could be considered (Ghita, 2009). In addition, these kinds of information could help the manufacturer to improve the CT machine, so that the benefit to risk ratio would be as high as reasonably achievable (Kalender, 2014). Considering this issue, dosimetry tools that accurately quantify the dose to children would improve administration of CT scans (making informed decision) of the pediatric body (Gu et al., 2009).

To obtain organ dose in a human body, two different approaches are possible: experimental procedures and Monte Carlo simulation. It was reported that Monte Carlo simulation is the most reliable way to obtain accurate values of dose 
under CT imaging (Caon et al., 2000; DeMarco et al., 2005; Lee et al., 2011) because the pediatric bodies lying on a CT table and X-ray beam are fully simulated, so that with an appropriate simulation model, the results are solid.

Up to now, phantoms with realistic anatomies were developed. BABY and CHILD developed by Zankl et al. (1995) and ADELAID a 14-year old phantom developed by Caon et al. (2000) were used in CT dosimetry calculation. Researchers at the University of Florida reported more comprehensive pediatric voxel phantoms (Lee et al., 2005, 2006). Lee et al. determined absorbed dose to organs of pediatric UF voxel phantoms (Lee et al., 2007). Recently, they published their results for reference hybrid pediatric phantoms (Lee et al., 2012). Despite the existence of reference data for received dose, these data are still limited because organ doses of the patients vary according to patient sizes, CT scanner model and scan protocols (Akhlaghi et al., 2014). So, there is a critical need to update and expand these data to provide more accurate estimation of cancer risk and patient-specific reporting of organ dose to the patient's electronic medical charts at the time of examination (Bolch et al., 2010).

As a result of this debate, the purpose of this study was to expand data for all whole-body non-reference pediatric voxel phantoms and to investigate the effect of their anatomical discrepancies (with reference values) in organ absorbed dose and effective dose. In this study, the relative differences between doses of reference and non-reference pediatric phantoms would be estimated and the possibility of finding a reasonable relationship between dose and anatomical data would be discussed. Finally, the uncertainty in amount of doses of non-reference phantoms, using those of reference phantoms, would be studied.

\section{Materials and methods}

\subsection{Monte Carlo dose calculation}

The Monte Carlo method is one of the most often used, accurate techniques for particle transport simulation, which can provide the amount of dose from particle interactions in the human body. In this study, MCNP4C, developed by the Los Alamos National Laboratory, was used for photon transport. Assuming charged particle equilibrium (CPE), absorbed dose was approximated by collision kerma and was recorded using the type 6 (F6: p) (track length estimate of photon energy deposition). The simulations provide dose in $\mathrm{MeV}_{\mathrm{g}}{ }^{-1}$, i.e. energy deposition $(\mathrm{MeV})$ per unit mass $(\mathrm{g})$, per emitted particle. Considering that the unit of absorbed dose is Gy $\left(\mathrm{J}_{\mathrm{kg}} \mathrm{kg}^{-1}\right)$, the output of the program should be multiplied by $1.6 \times 10^{-7}$. Since MCNP provides absorbed dose per simulated photon, total absorbed doses were calculated based on total photon emitted from the source. For calculation of red bone marrow (RBM) absorbed dose, F4 tally was used. This tally provides the volume flux in unit of $\mathrm{cm}^{-3}$. Therefore, the outcomes of this tally are multiplied by the flux to absorbed dose coefficients $\left(\mathrm{mGy} . \mathrm{cm}^{3}\right)$, to obtain RBM dose in $\mathrm{mGy}$. These coefficients are determined by $\mathrm{dE}$ (energy) and $\mathrm{dF}$ (flux to absorbed dose coefficient in that energy) cards in the input of program. In all

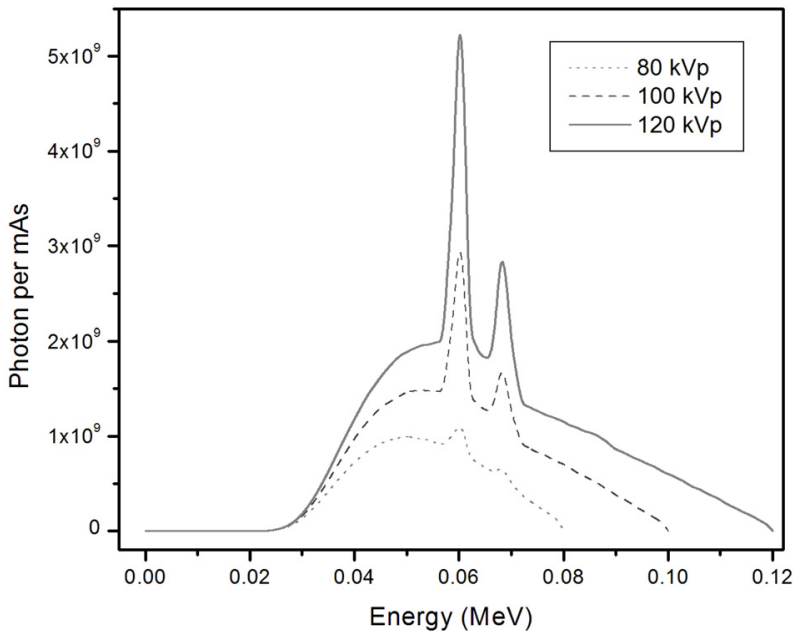

Fig. 1. X-ray spectra of Siemens Somatom Sensation 16 at tube voltages 80,100 and $120 \mathrm{kVp}$.

simulations, the number of histories was selected to obtain statistically meaningful results with relative errors less than to $2 \%$ in most organs located in the scan field.

\subsubsection{Effective dose}

Effective dose is the tissue-weighted sum of the equivalent doses (radiation weighted of absorbed doses) in all specified tissues and organs of the body. The effective dose is the protection quantity, the main use of which is the prospective dose assessment for planning and optimization in radiological protection (ICRP, 2007b). Although the effective dose quantity is based on reference phantoms chosen by convention, this does not mean that one cannot use the effective dose quantity if other phantoms have been chosen (Karimi Shahri et al., 2013). In addition, in this study only gender-specific organs are considered to yield either the male- or female effective dose of a given gender. They are provided strictly for relative comparison of phantom anatomy at each age group (Lee et al., 2007), as true values of effective dose are gender-averaged quantities.

\subsection{CT scanner simulation}

A Siemens Somatom Sensation 16 (Siemens Medical Systems, Germany) was simulated within the MCNP4C Monte Carlo radiation transport code. The $\mathrm{CT}$ scanner had a fan beam originating from the focal spot with a fan beam angle of $52^{\circ}$ and a focal spot-to-axis distance of $57 \mathrm{~cm}$. The information about X-ray spectra and scanner's characteristics were provided by manufacturer. Figure 1 displays X-ray spectra at tube voltages of 80,100 , and $120 \mathrm{kVp}$.

In the MCNP code, there are at least three ways to define the specific shape of the fan beam (Khursheed et al., 2002; Gu et al., 2008, 2009). In this research, the same method as Khursheed et al. was used. Therefore, CT imaging was simulated by exposing a series of contiguous transverse slices of $1 \mathrm{~cm}$ thickness to emit X-rays from sources lying on a circle around the phantom (Khursheed et al., 2002). 


\subsection{Validation of CT beam model}

A common way to validate the CT scanner model is through comparisons between experimentally measured and simulated computed tomography dose index (CTDI) values (Jarry et al., 2003; Staton et al., 2006; Gu et al., 2009; Lee et al., 2011, 2012). For this purpose, a set of CTDI data was simulated for head and body CTDI phantoms with diameters of 16 and $32 \mathrm{~cm}$, respectively, and compared with the measured CTDI values reported by Lee et al. (2012), under the same radiation exposure condition. To perform the comparison, the CTDI phantoms were modeled as a cylinder having a diameter of $16 \mathrm{~cm}$ for head and $32 \mathrm{~cm}$ for body phantoms, with a length of $15 \mathrm{~cm}$ each. The material composition of the CTDI phantoms was simulated as polymethylmethacrylate with a density of $1.19 \mathrm{~g} . \mathrm{cm}^{-3}$. The Radical ion chamber was modeled as three $10 \mathrm{~cm}$ long concentric cylinders. The innermost cylinder with a diameter of $6.7 \mathrm{~mm}$ defined the active air volume. The second cylinder with a diameter of $10.2 \mathrm{~mm}$ defined the chamber wall which is C552 air-equivalent material with a density of $1.76 \mathrm{~g} . \mathrm{cm}^{-3}$. The third cylinder with a diameter of $13.7 \mathrm{~mm}$ defined a build-up cap, which was modeled as polyacetal plastic with a density of 1.43 g.cm ${ }^{-3}$ (Lee et al., 2011).

\subsection{Computational model of anthropomorphic phantoms}

\subsubsection{GSF tomographic pediatric models}

Zankl et al. published the first voxelized tomographic models in 1988, where they created two different phantoms from CT data of two female Caucasian children (Zankl et al., 1988). The first model, called BABY, was developed from a wholebody scan of an 8-week-old female cadaver. The second, called CHILD was created from a 7-year-old female child undergoing whole-body radiation therapy for leukemia treatment. In this study, BABY-new (new model of BABY with some refinements done by Zankl et al.) and CHILD were used for dose estimations. The resolutions of voxel models of BABY and CHILD were $0.85 \times 0.85 \times 4 \mathrm{~mm}^{3}$ and $1.54 \times 1.54 \times$ $8 \mathrm{~mm}^{3}$, respectively (German Research Center for Environmental Health, 2014).

\subsubsection{Virtual population of pediatric phantoms}

The Virtual Population Program at the Foundation for Research on Information Technologies in Society (IT'IS) in Switzerland created numerical human models representing the entire population (Christ et al., 2010; IT'IS foundation, 2012). Currently, the Virtual population includes eight available highly detailed anatomical whole-body models of adults and children of both genders. These phantoms were created based on high-resolution MR images of healthy volunteers. Each model was reconstructed as a three-dimensional object with approximately 80 high-resolution organs and tissues (Christ et al., 2010). The resolution of voxel format used in this study was $5 \times 5 \times 5 \mathrm{~mm}^{3}$. Gender, age, height, weight and number of tissues of pediatric phantoms were listed in Table 1.
Table 1. Specifications of pediatric voxel phantoms developed at IT'IS Foundation.

\begin{tabular}{cccccc}
\hline Name & Gender & $\begin{array}{c}\text { Age } \\
(\text { year })\end{array}$ & $\begin{array}{c}\text { Height } \\
(\mathbf{c m})\end{array}$ & $\begin{array}{c}\text { Weight } \\
(\mathbf{k g})\end{array}$ & $\begin{array}{c}\text { No. of } \\
\text { tissues }\end{array}$ \\
\hline Roberta & Female & 5 & 109 & 17.8 & 66 \\
Thelonious & Male & 6 & 117 & 19.3 & 76 \\
Eartha & Female & 8 & 136 & 30.7 & 75 \\
Dizzy & Male & 8 & 139 & 26.0 & 66 \\
Billie & Female & 11 & 147 & 35.4 & 75 \\
Louis & Male & 14 & 169 & 50.4 & 77 \\
\hline
\end{tabular}

\subsubsection{UF Series B pediatric voxel phantom}

The reference voxel phantoms of this study were those of the UF Series B phantoms of five children: 9-month male (height of $69.75 \mathrm{~cm}$ and weight of $8.87 \mathrm{~kg}$ ), 4-year female (height of $100.75 \mathrm{~cm}$ and weight of $16.65 \mathrm{~kg}$ ), 8-year female (height of $126.4 \mathrm{~cm}$ and weight of $28.41 \mathrm{~kg}$ ), 11-year male (height of $143.8 \mathrm{~cm}$ and weight of $33.59 \mathrm{~kg}$ ), and 14-year male (height of $161.2 \mathrm{~cm}$ and weight of $48.8 \mathrm{~kg}$ ) (Goorley, 2007). The UF Series B phantoms were developed from their predecessor UF Series A phantoms, which were in turn constructed through image segmentation of head CAP CT scans of patients. These phantoms are not patient specific and their body sizes and masses were closely aligned to that of ICRP Publication 89 (ICRP, 2002). Due the fact that the ages of this phantom series did not match with those of nominal ICRP reference ages, the organ masses and height/weight were taken as interpolated values from ICRP Publication 89 in that series (Lee, 2006).

Note that UF pediatric series are representative of reference pediatric subjects but BABY, CHILD (Zankl et al., 1988) and virtual phantoms of IT'IS Foundation are not in 50th percentile of all children (worldwide) (Christ et al., 2010). Table 2 contains the reference values of organs masses reported in ICRP Publication 89 (ICRP, 2002) and organ masses of these non-reference phantoms.

\subsection{Investigations on anatomical differences}

For a more detailed study on organ dose discrepancies between phantoms, anatomical differences in the scan field should be reviewed. The depth below the surface is a parameter that significantly influences the dose from external radiation. They indicate the amount of overlying tissues, by which each point of an organ or tissue is shielded from radiation impinging from X-ray source rotating around the body. Thus, depth distributions below the body surfaces were calculated for main organs of these phantoms. The distributions have been evaluated for 1 million points sampled randomly in the organs considered (ICRP, 2009).

\section{Results}

\subsection{Model validation}

Four different point doses (central dose and doses at 12, 3, and 6 o'clock positions) were determined within the CTDI 
Table 2. Reference values for masses of organs/tissues reported in ICRP Publication 89 in comparison with those of non-reference phantoms of this study.

\begin{tabular}{|c|c|c|c|c|c|c|c|c|c|c|c|c|c|}
\hline & Newborn & 1 year & 5 years & 10 years & 15 years $(M)$ & BABY & CHILD & Roberta & Thelonious & Eartha & Dizzy & Billie & Louis \\
\hline Adrenals & 6 & 4 & 5 & 7 & 10 & 4 & 4 & - & 3 & 2.5 & - & 8 & 2.5 \\
\hline Salivary glands & 6 & 24 & 34 & 44 & 68 & - & - & - & - & - & - & - & - \\
\hline Stomach wall & 7 & 20 & 50 & 85 & 120 & 6.5 & 52 & 66 & 142 & 121 & 113 & 443 & 127 \\
\hline SI wall & 30 & 85 & 220 & 370 & 520 & 31 & $485^{\mathrm{a}}$ & 146 & 60 & 269 & 49 & 400 & 314 \\
\hline Colon wall & 17 & 50 & 120 & 210 & 300 & 23 & 84 & 130 & 230 & 256 & 227 & 280 & 349 \\
\hline Liver & 130 & 330 & 570 & 830 & 1300 & 182 & 732 & 495 & 570 & 717 & 880 & 880 & 1265 \\
\hline Gall bladder & 3.3 & 9.4 & 17.6 & 30.4 & 52.7 & 2.5 & - & 6.5 & 8 & 5 & 12 & 20 & 22 \\
\hline Pancreas & 6 & 20 & 35 & 60 & 110 & 3 & 30 & 8 & 11 & 21 & 10 & 25 & 27 \\
\hline Brain & 380 & 950 & $1310 / 1180$ & $1400 / 1220$ & 1420 & 332 & 1400 & 1246 & 1310 & 1410 & 1286 & 1250 & 1620 \\
\hline Heart wall & 20 & 50 & 85 & 140 & 230 & $39^{a}$ & $206^{a}$ & $53^{a}$ & $248^{a}$ & $155^{\mathrm{a}}$ & $256^{\mathrm{a}}$ & $157^{\mathrm{a}}$ & $431^{\mathrm{a}}$ \\
\hline Lungs & 60 & 150 & 300 & 500 & 900 & 41 & 198 & 315 & 348 & 451 & 509 & 542 & 700 \\
\hline Spleen & 9.5 & 29 & 50 & 80 & 130 & 14 & 150 & 90 & 120 & 147 & 285 & 150 & 321 \\
\hline Thymus & 13 & 30 & 30 & $40 / 35$ & 35 & 11 & 30 & 21 & 30 & 9 & 30 & 30 & 24 \\
\hline Thyroid & 1.3 & 1.8 & 3.4 & 7.9 & 12 & 1.1 & 4.9 & - & - & - & - & 11 & 7.5 \\
\hline Kidneys & 25 & 70 & 110 & 180 & 250 & 30 & 188 & 106 & 140 & 165 & 160 & 170 & 272 \\
\hline Urinary bladder & 4 & 9 & 16 & 25 & 40 & 2.5 & 24 & $26^{\mathrm{a}}$ & $32^{\mathrm{a}}$ & $145^{\mathrm{a}}$ & $53^{\mathrm{a}}$ & $38^{\mathrm{a}}$ & $196^{\mathrm{a}}$ \\
\hline Testes & 0.85 & 1.5 & 1.7 & 2 & 16 & - & - & - & 3 & - & 1 & - & 17 \\
\hline Prostate & 0.8 & 1 & 1.2 & 1.6 & 4.3 & - & - & - & 3 & - & 4 & - & 12 \\
\hline Ovaries & 0.3 & 0.8 & 2 & 3.5 & - & 0.4 & 2.6 & 1.5 & - & 3.9 & - & 4.7 & - \\
\hline Uterus & 4 & 1.5 & 3 & 4 & - & 1.3 & 14 & 5.5 & - & 21 & - & 23 & - \\
\hline Total body (kg) & 3.5 & 10 & 19 & 32 & 56 & 4.2 & 21.7 & 17.8 & 19.3 & 30.7 & 26 & 35.4 & 50.4 \\
\hline
\end{tabular}

${ }^{a}$ The wall and content was not segmented independently.

Table 3. Scan parameters considered in the simulations.

\begin{tabular}{|c|c|c|c|c|c|c|c|}
\hline \multirow{3}{*}{$\begin{array}{c}\text { Scan } \\
\text { parameters }\end{array}$} & \multirow{2}{*}{$\begin{array}{c}\text { Tube voltage } \\
(\mathbf{k V p})\end{array}$} & \multirow{2}{*}{$\begin{array}{l}\text { Collimation } \\
\text { (mm) }\end{array}$} & \multirow[t]{2}{*}{ Pitch } & \multicolumn{4}{|c|}{ Scan coverage } \\
\hline & & & & Head & Chest & Abdomen-Pelvis & CAP \\
\hline & $\begin{array}{c}80,100,120 \\
\text { and } 140\end{array}$ & 10 & 1 & $\begin{array}{l}\text { From top of the } \\
\text { head to the } 2 \text { nd } \\
\text { cervical vertebra }\end{array}$ & $\begin{array}{l}\text { From the } \\
\text { clavicles to the } \\
\text { middle of the liver }\end{array}$ & $\begin{array}{l}\text { From the top of } \\
\text { liver to the } \\
\text { midfemoral head }\end{array}$ & $\begin{array}{c}\text { From the } \\
\text { clavicles to the } \\
\text { midfemoral head }\end{array}$ \\
\hline
\end{tabular}

head and body phantoms by using the ion chamber with the collimation of $10 \mathrm{~mm}$ under the three tube potentials of 80 , 100 , and $120 \mathrm{kVp}$. The weighted CTDI $\left(\mathrm{CTDI}_{\mathrm{w}}\right)$, which is defined as the summation of one-third of CTDI center $_{\text {and two- }}$

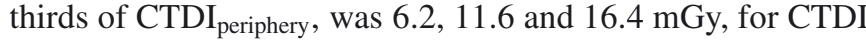
head phantom at tube voltages of 80,100 and $120 \mathrm{kVp}$, respectively. The simulated doses of this study agreed with the measured ones with maximum error of almost $9 \%$ for all tube potentials. These results were comparable with those given in other published studies (Jarry et al., 2003; Staton et al., 2006; Lee et al., 2011, 2012).

\subsection{Organ dose estimations}

Organ absorbed dose for almost 30 organs were calculated for BABY, CHILD, UF series B and IT'IS foundation voxel phantoms at head, chest, abdomen-pelvis, and CAP examinations. The scan protocols were generic and were in good agreement with others available in the literature (Lee et al., 2012). Table 3 summarizes the scan protocols used in this study. Considering the linear relationship between the mAs and dose (Akhlaghi et al., 2014), the results were normalized to mAs. Figure 2 displays some major organ absorbed doses in unit $\mathrm{mGy} / \mathrm{mAs}$ at $80 \mathrm{kVp}$ for pediatric series of IT'IS Foundation. Moreover, the doses absorbed by main organs in CAP scan at tube voltage of $120 \mathrm{kVp}$ were presented in Table 4.

According to the results, brain in head scan, lungs in chest scan and colon, kidneys and bladder in abdomen-pelvis scan, which were exposed directly in the scan range, received the maximum absorbed doses. In the head scan, absorbed doses of brain and eye lenses were between $7.2 \times 10^{-2}$ to $9.7 \times 10^{-2} \mathrm{mGy} / \mathrm{mAs}$ and $7.8 \times 10^{-2}$ to $9.4 \times 10^{-2} \mathrm{mGy} / \mathrm{mAs}$, respectively. The maximum value of brain dose in other scan ranges was $15 \times 10^{-3}, 6.0 \times 10^{-4}$, and $21 \times 10^{-3} \mathrm{mGy} / \mathrm{mAs}$, for chest, abdomen-pelvis and CAP examinations, respectively.

In chest examination, lungs, thymus, and heart-wall received high doses relative to the other organs. The absorbed doses of lungs and heart-wall ranged from $6.9 \times 10^{-2}$ to $9.0 \times 10^{-2} \mathrm{mGy} / \mathrm{mAs}$ and $6.9 \times 10^{-2}$ to $9.2 \times 10^{-2} \mathrm{mGy} / \mathrm{mAs}$, respectively. The small intestine-wall, kidneys, bladder and colon-wall receives relatively high doses, which ranged from $7.8 \times 10^{-2}$ to $10.3 \times 10^{-2} \mathrm{mGy} / \mathrm{mAs}$, from $7.7 \times 10^{-2}$ to $10.6 \times$ $10^{-2} \mathrm{mGy} / \mathrm{mAs}$, from $6.6 \times 10^{-2}$ to $10.9 \times 10^{-2} \mathrm{mGy} / \mathrm{mAs}$, 


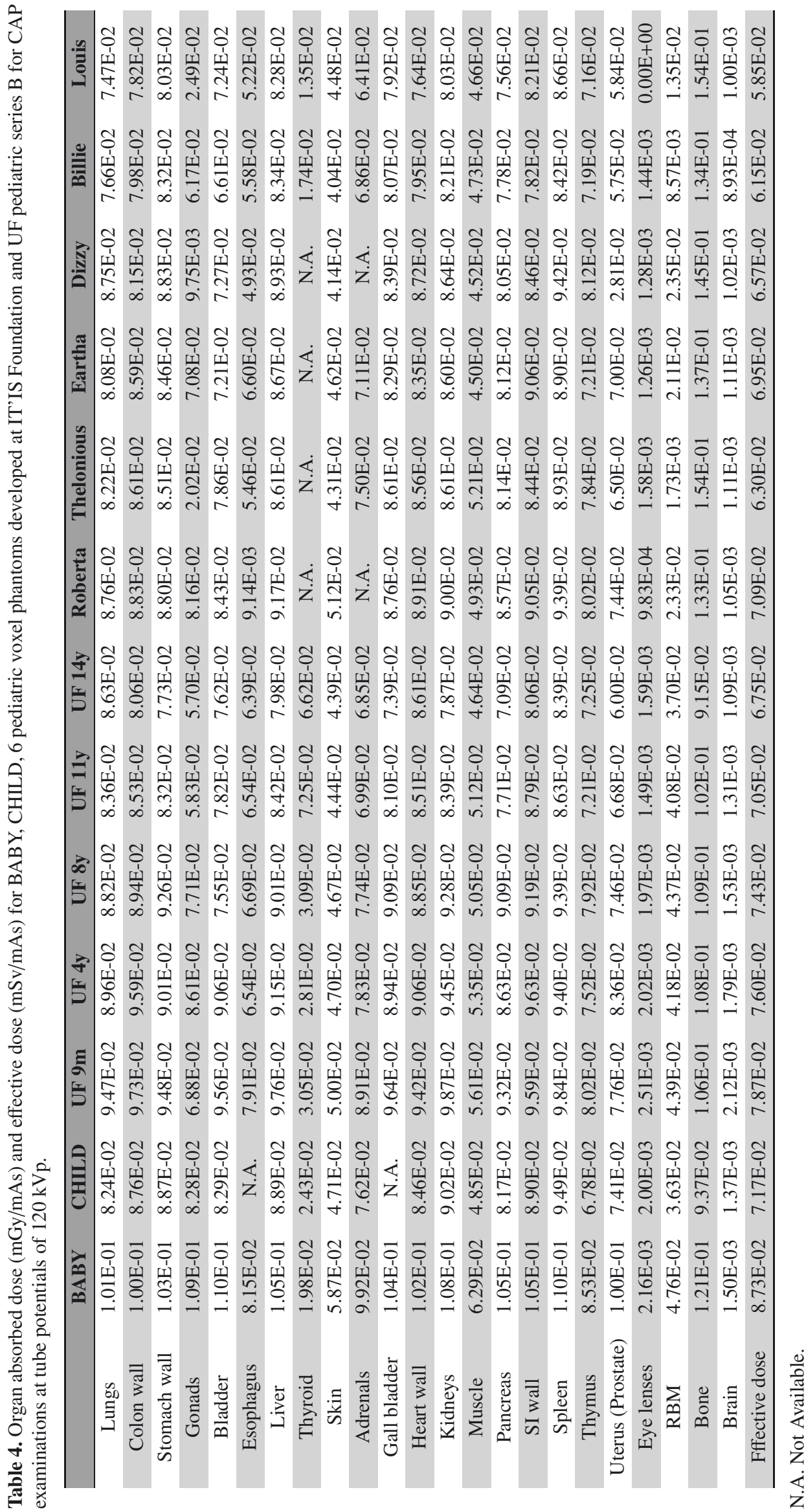



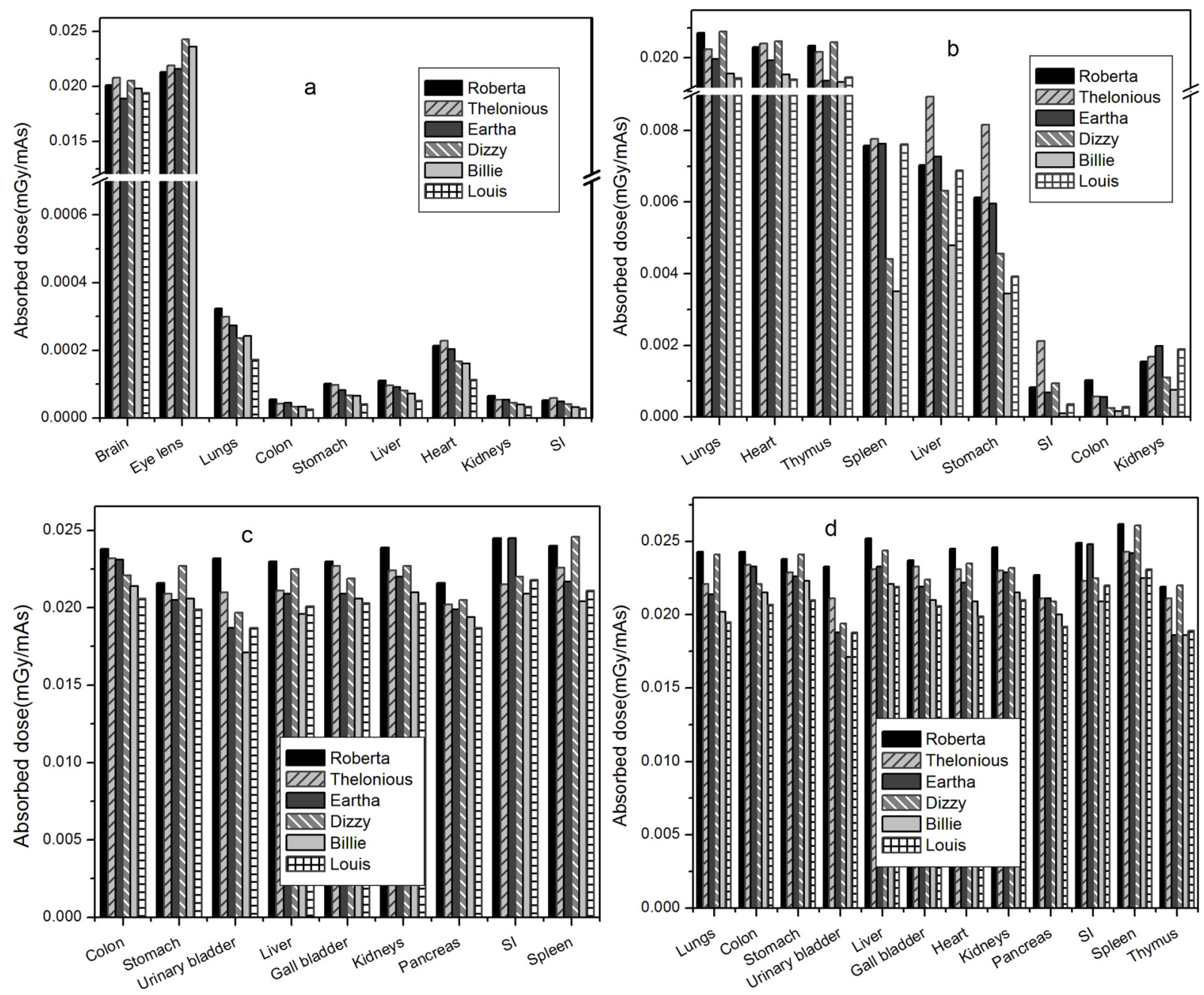

Fig. 2. Organ absorbed dose in mGy/mAs for IT'IS pediatric phantoms for scans of (a) head, (b) chest, (c) abdomen-pelvis and (d) CAP at tube voltage of $80 \mathrm{kVp}$.

and from $7.8 \times 10^{-2}$ to $9.9 \times 10^{-2} \mathrm{mGy} / \mathrm{mAs}$, respectively at abdomen-pelvis study.

\subsection{Effective dose estimations}

The values of effective dose based on ICRP Publication 103 weighting factors were tabulated in Table 5 at tube voltage of $120 \mathrm{kVp}$. In constant tube current, for all phantoms and scan ranges, the effective dose at higher tube voltages had higher amounts (e.g. effective dose at $140 \mathrm{kVp}$ was $\sim 500 \%$ higher than that at $80 \mathrm{kVp}$ ).

\subsection{Distributions of depths of main organs/tissues}

Depth distributions of some main organs located in the scan range were calculated for all phantoms. Figure 3 displays the depth distribution of brain, colon and lungs for phantoms with the similar ages. The same data are available for other main organs of all phantoms. According to the results, on average, brain of UF 9-month, lungs of CHILD, colons of Billie and Louis, compared to their peers, are seated deeper in the body.

\section{Discussion}

Given the results of organ dose estimations, when scan range did not cover the organ directly, as distance between them increased, the organ absorbed dose and its contribution in total dose decreased, as expected. In the head scan, lungs were not covered directly, so the maximum value of lungs dose was almost 30 times less than that at chest scan and therefore it had a small contribution in total effective dose.

In the UF voxel phantoms, as age increases, the effective dose decreases; but the same trend was not observed for virtual phantoms of IT'IS Foundation. This could be due to the fact that anatomical data of these non-reference phantoms have not been adjusted based on ICRP Publication 89. Namely, they have many anatomical differences (e.g. organ location, organ size, organ mass) with 50th percentiles children of their own age (Tab. 2). For instance, considering Figure 3 and the results of dose estimations, the colon tissues of BABY (compared to UF 9-month), UF 11-year (compared to Billie) and UF 14-year (compared to Louis), UF 4-year (compared to Roberta, Thelonious and CHILD) are located closer to the body surface, so they received more amount of dose, as expected. There were 
similar trends for the brain and lungs tissues of these phantoms, so that the one, which was located deeper, received less amount of dose. Therefore, the discrepancies between doses delivered to other organs can also be justified considering their sizes, their locations and the amount of overlaying tissues located in the beam path. For a given phantom, the contribution of organ doses in effective dose depends on the scan coverage, and organs' type and location in human anatomy. The organs, which received the maximum absorbed doses, have a greater share in effective dose (e.g. lung in chest scan, brain in head scan, and SI, stomach, colon and bladder in abdomen-pelvis imaging) while, the organs outside the scan coverage have little contributions in effective dose except for chest scan. This is due to the low attenuation of X-ray radiations in air, which have filled the lungs and the fact that lungs are in the middle of sensitive organs/tissues, so in chest examination when they covered fully in scan region, they scatter the radiations to the other sensitive organs.

\subsection{Age dependent organ absorbed doses}

Organ doses were compared between reference and nonreference pediatric phantoms to find a relation between age and organ doses. As known, when desired organs are fully covered within the scan range, due to the higher attenuations of overlaying tissues of older phantoms, their internal organs would be more protected, so that they receive lower organ doses (Lee et al., 2012). However, for the non-reference phantoms of IT'IS Foundation this does not happen usually which is the result of models' limitations, such as insufficiency of image quality of digestive tract to be segmented completely, not including some tissues like salivary glands in all phantoms, and thyroid, adrenals in some phantoms, not separating the wall and content of some organs like bladder, etc (Christ et al., 2010). In addition, this could be the results of their discrepancies with reference anatomical data (e.g. differences in the depth distribution of organs).

At $120 \mathrm{kVp}$ in head scan, the maximum relative difference between brain doses of group 1 (BABY and UF 9-month), group 2 (CHILD, UF 4-year old, Roberta, and Thelonious), group 3 (UF 11-year old and Billie), and group 4 (UF 14-year old and Louis) were 14\%, 7\%, 6\%, and $0.3 \%$, respectively. In chest scan their maximum differences in lung doses were $\sim 6 \%, \sim 14 \%, \sim 8 \%$ and $\sim 15 \%$, respectively, while their maximum discrepancies between colon doses in abdomen-pelvis imaging was about $4 \%, 10 \%, 6 \%$ and $2 \%$, respectively. Can be said that for organs located in scan regions, which have the maximum contribution in effective dose, the value of absorbed doses would be estimated from our obtained results with error less than $15 \%$

The absorbed doses of UF-8-year, Dizzy and Eartha (group 5) in some major organs (in $\mathrm{mGy} / \mathrm{mAs}$ ) for all scan ranges at $80 \mathrm{kVp}$ were displayed in Figure 4. Although, there are many discrepancies in their anatomical data but the relative differences in absorbed doses of most organs located in the scan range were less than $10 \%$. Moreover, it was observed that as distance between organ and scan range increased, the relative differences enhanced. 

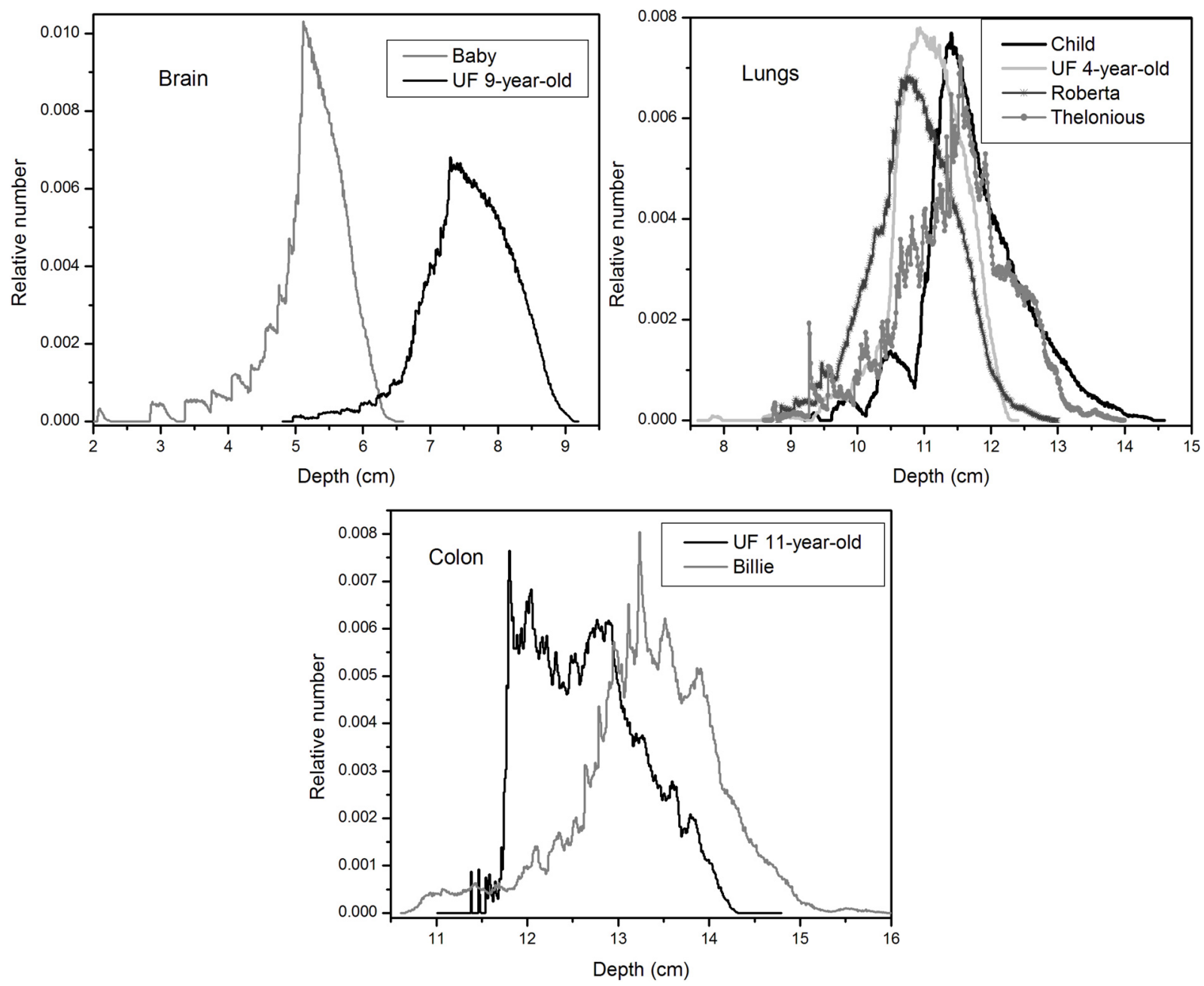

Fig. 3. Comparisons of brain, lungs and colon depth distributions of pediatric phantoms with similar ages.

Considering UF 8-year old as a reference phantom in this age, the relative differences between main organs absorbed doses of Dizzy and Eartha and that of UF were calculated for all scan ranges and tube potentials. The relative differences between organ doses of the Dizzy and Eartha relative to UF 8 -year at $80 \mathrm{kVp}$ were plotted in Figure 5.

In abdomen-pelvis study at $80 \mathrm{kVp}$, the relative differences for most of the organs included in scan range were less than $10 \%$ (e.g. colon, bladder, liver, kidneys, and spleen). For some organs such as small intestine-wall, gall bladder and stomachwall this discrepancies were slightly more than $10 \%$. This could be due to different shielding effects of other organs. The relative differences for main organs, which were away from the scan range, were up to $27 \%$ (brain). Such a trend was almost observed in the other scan regions. The level of disagreements depends on organ type and its relative position in human anatomy.

In these comparisons, phantoms were classified according to their ages. Considering the maximum relative differences less than $15 \%$, one could generalize the outcomes to the nonreference children with the same age group.

\subsection{Comparison of age-based effective doses}

Significant discrepancies in effective doses were observed between different voxel phantoms with the similar ages. The minimum and maximum relative differences in effective dose for group 1 were $\sim 11 \%$ (in CAP examination) and $\sim 25 \%$ (in head examination), respectively, while minimum and maximum relative differences in effective dose for group 2 were $0.65 \%$ (in head scan) and $43 \%$ (in chest scan). In addition, the minimum and maximum relative differences for group 3 were $\sim 7 \%$ (in head examination) and $\sim 33 \%$ (in chest examination), respectively. The minimum and maximum relative differences for group 4 were $\sim 6 \%$ (in abdomen-pelvis examination) and $\sim 47 \%$ (in head examination), respectively.

Considering Figure 5 for group 5, in abdomen-pelvis study, in spite of all anatomical discrepancies, the effective dose delivered to Dizzy $\left(1.35 \times 10^{-2} \mathrm{mSv} / \mathrm{mAs}\right)$, Eartha $(1.37 \times$ $\left.10^{-2} \mathrm{mSv} / \mathrm{mAs}\right)$ and UF 8 -year old $\left(1.3 \times 10^{-2} \mathrm{mSv} / \mathrm{mAs}\right)$ showed significant agreement with a relative difference of about $5 \%$. This means that the organs located out of scan coverage had low contributions in effective dose (e.g. $\sim 0.7 \%$ in Earths's heart-wall). Greater disagreements were observed 

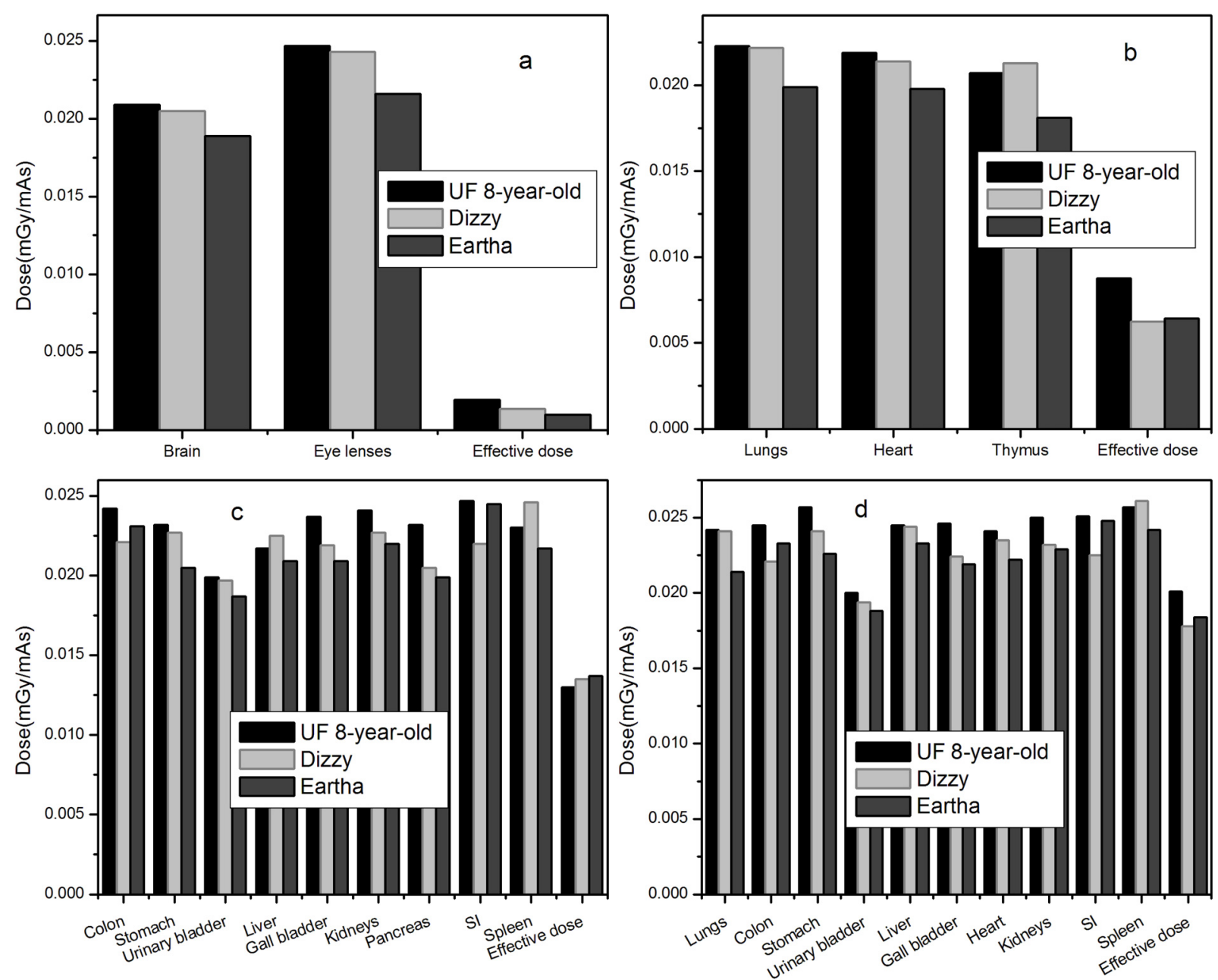

Fig. 4. Comparison of organ absorbed dose in $\mathrm{mGy} / \mathrm{mAs}$ for scans of (a) head, (b) chest, (c) abdomen-pelvis and (d) CAP for 8-year phantoms at tube voltage of $80 \mathrm{kVp}$.

between effective doses in other examinations (e.g. $29 \%$ at tube voltage of $80 \mathrm{kVp}$ in chest scan). This is due to the fact that, in the chest and head scans, limited numbers of directly exposed organs, which have high tissue weighting factors are located in the scan range (brain in head scan, and lungs and heart in chest scan). This means that, although, the discrepancies between the most organs covered in scan range (heart and lung) were less than $10 \%$ and lungs dose contribute in $51 \%$ and $45 \%$ of total effective dose in Dizzy and Eartha, respectively, but because of the $50 \%$ share in effective dose by organs out of the scan coverage (with high relative differences) the disagreements increased. The minimum value of effective dose and maximum value of relative difference were observed at head scan. The $\sim 50 \%$ discrepancy between UF and Eartha is due to their considerable differences in the amount of RBM located in the skull and mandible.

Although, the same anatomical landmarks were used to define the scan ranges of all phantoms, but different portions of the organs were included in a given scan range because of the discrepancies between anatomical data of non-reference and reference phantoms. So, it was difficult to find consistent differences in effective dose between these phantoms except for CAP imaging. As mentioned, when organs are included in scan range, the relative differences between doses received by phantoms decreased. Therefore, in CAP examination, in which most of sensitive organs are fully covered, these differences in effective doses diminished.

According to the results of these two sections, the total number of phantoms in each age group is not sufficient to make a general conclusion for age-based effective dose for all scan ranges except for CAP examinations. So, by using a greater number of voxel-based phantoms, one may generalize the effective doses for children with similar ages.

\subsection{Weight- and height-based comparison of effective doses}

Considering the assumption that height and weight are responsible for magnitude of effective dose received by the patients, the authors wondered if they could generalize current data to the other groups of non-reference pediatrics. In Table 5, the height, weight and body mass index (BMI) of all phantoms together with the total effective doses in head, chest, abdomen-pelvis and CAP scans at tube voltage of $120 \mathrm{kVp}$ 

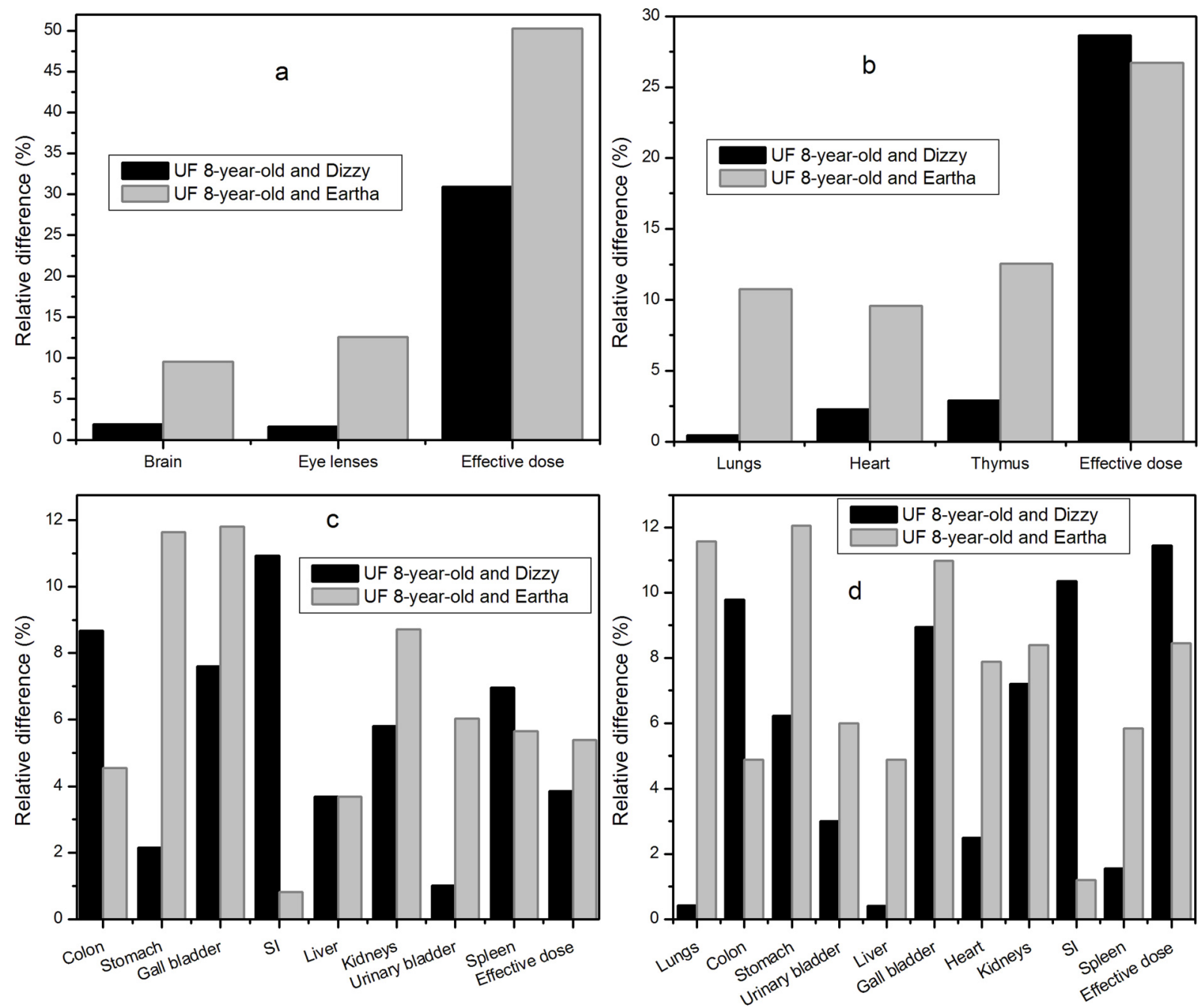

Fig. 5. Relative differences of organ dose in percent for scans of (a) head, (b) chest, (c) abdomen-pelvis and (d) CAP for 8-year phantoms at tube voltage of $80 \mathrm{kVp}$.

were tabulated. BMI is a numeric measure of person thinness or thickness. According to Table 5, the values of BMI suggest that there is a great variability between the relative weights of similar phantoms.

In Figure 6, the relative differences between effective doses of two phantoms series were presented. According to Figure 6 and Table 5, Roberta and CHILD, which have similar heights, their effective doses matched in abdomen-pelvis and CAP scans. While, for Thelonious and CHILD, which have the same sizes, good compatibilities were only obtained in head scans. Weights of Dizzy and UF 8-year are almost the same, but their effective doses agreed in abdomen-pelvis and CAP scans. The similar trend was observed for Louis and UF 14-year. For UF 11-year and Billie, which have the same sizes and BMI values, more consistency were observed between the results, so that their effective doses in head, abdomen-pelvis and CAP imaging were in good agreements. It seems that height and weight together with the value of BMI are more appropriate indicators than age for investigating the relationship between phantom doses. Good agreements between these phantoms were only observed in CAP scan, which covers directly most of sensitive

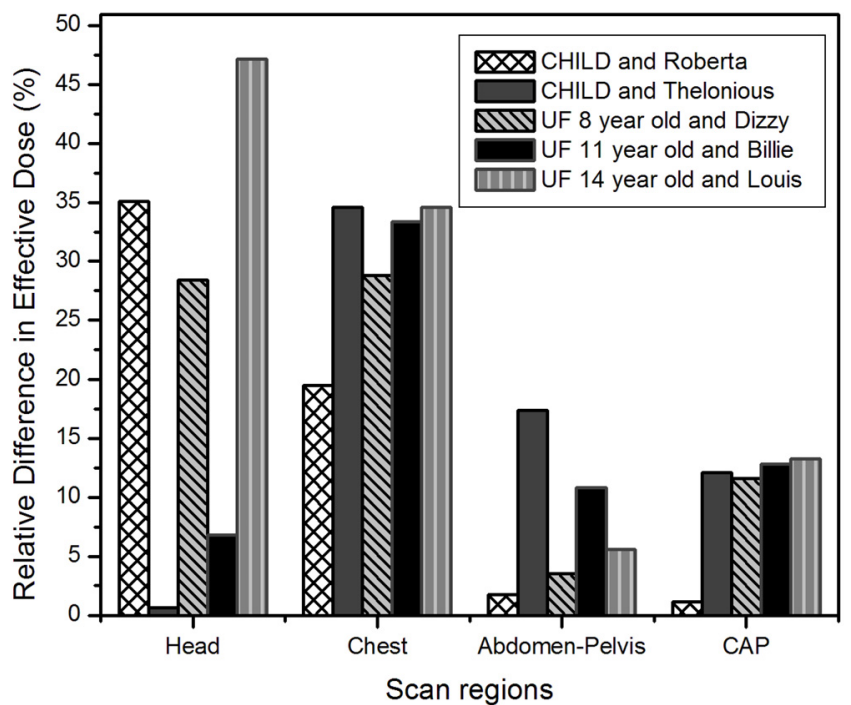

Fig. 6. Relative differences of effective dose in percent for scans of head, chest, abdomen-pelvis and CAP for three phantom series at tube voltage of $120 \mathrm{kVp}$. 
organs and differences in physical properties of tissues have less effect in radiation doses. Can be said that these datasets are not enough for an accurate and logical conclusion (with error less than $10 \%$ ) for the other scan regions. So, to find whether there is a relationship between body size and mass with effective dose or not, and to generalize the results to the other children, which are not in 50th percentile height and weight, a larger group of pediatric phantoms would be needed.

\section{Conclusion}

The organ doses and effective doses were estimated for reference and non-reference pediatric phantoms undergoing CT examinations using Monte Carlo simulations. Organ doses for head, chest, abdomen-pelvis and CAP examinations were calculated and then compared with each other. Considering the facts that anatomical data of non-reference phantoms have not be adjusted based on ICRP Publication 89, sometimes significant differences in estimated doses were observed between the results of phantoms with similar ages. However, it was demonstrated that for organs exposed directly, the values of absorbed dose of each age group could be estimated and generalized to children in the same age group. In addition, because of the limited number of phantoms, the attempt to find a possible relationship between effective dose and phantoms' height/weight for all scan ranges was not successful except in CAP scans. In the future study, the organ dose estimation will be extended to cover various ages, heights and sizes of non-reference pediatrics. Incorporating this library of phantoms with different sizes into the database would help in individualizing the organ dose and effective dose calculations.

Acknowledgements. The authors would like to acknowledge Dr. Karl Stierstorfer for his cooperation in providing the X-ray spectra of Siemens Somatom Sensation 16 scanner.

\section{References}

Akhlaghi P., Miri Hakimabad H., Rafat Motavalli L. (2014) An overview of exposure parameters, dose measurements and strategies for dose reduction in pediatric CT examinations, Radioprotection 49, 9-15.

Berrington de Gonzàlez A., Mahesh M., Kim K.P., Bhargavan M., Lewis R., Mettler F., Land C. (2009) Projected cancer risks from computed tomographic scans performed in the United States in 2007, Arch. Intern. Med. 169, 2071-2077.

Bolch W.E., Lee C., Lee C., Hurtado J., Williams J.L. (2010) Applications to computed tomography for pediatric patients. In: Handbook of anatomical models for radiation dosimetry (X.G. Xu, K.F. Eckerman Eds) pp. 487-510. Taylor \& Francis, New York.

Brenner D.J. (2002) Estimating cancer risks from pediatric CT: going from the qualitative to the quantitative, Pediatr. Radiol. 32, 228-231.

Brenner D.J., Hall E.J. (2007) Computed tomography - an increasing source of radiation exposure, New Engl. J. Med. 357, 2277-2284.

Caon M., Bibbo G., Pattison J. (2000) Monte Carlo calculated effective dose to teenage girls from computed tomography examinations, Radiat. Prot. Dosim. 90, 445-448.
Christ A. et al. (2010) The Virtual Family - development of surfacebased anatomical models of two adults and two children for dosimetric simulations, Phys. Med. Biol. 55, 23-38.

DeMarco J.J., Cagnon C.H., Cody D.D., Stevens D.M., McCollough C.H., O’Daniel J., McNitt-Gray M.F. (2005) A Monte Carlo based method to estimate radiation dose from multidetector CT (MDCT): Cylindrical and anthropomorphic phantoms, Phys. Med. Biol. 50, 3989-4004.

German Research Center for Environmental Health (2014) http:// www.helmholtz-muenchen.de/amsd/service/scientific-services/ virtual-human-phantoms-download/index.html

Ghita M. (2009) Computer simulations to estimate organ doses from clinically validated cardiac, neuro, and pediatric protocol for multiple detectors computed tomography scanners, $\mathrm{PhD}$. thesis, University of Florida.

Goorley T. (2007) MCNP medical physics database, Los Alamos National Laboratory, LA-UR-07-2777, 1-18.

Gu J., Bednarz B., Xu X.G., Jiang S.B. (2008) Assessment of patient organ doses and effective doses using the VIP-MAN adult male phantom for selected cone-beam CT imaging procedures during image guided radiation therapy, Radiat. Prot. Dosim. 131, 431-443.

Gu J., Bednarz B., Caracappa P.F., Xu X.G. (2009) The development, validation and application of a multi-detector CT (MDCT) scanner model for assessing organ doses to the pregnant patient and the fetus using Monte Carlo simulations, Phys. Med. Biol. 54, 2699-2717.

ICRP Publication 89 (2002) Basic anatomical and physiological data for use in radiological protection reference values, Ann. ICRP 32, 1-277. Pergamon Press.

ICRP Publication 102 (2007a) Managing patient dose in multidetector computed tomography (MDCT) Ann. ICRP 37 (1), 1-79. Pergamon Press.

ICRP Publication 103 (2007b) The 2007 recommendations of the international commission on radiological protection, Ann. ICRP 37 (2-4), 1-328. Pergamon Press.

ICRP Publication 110 (2009) Adult reference computational phantoms, Ann. ICRP 39 (2), 1-162. Elsevier.

IT'IS Foundation for Research on Information Technologies in Society (2012) Anatomical models. http://www.itis.ethz.ch/ services/anatomical-models/overview/.

Jarry G., DeMarco J.J., Beifuss U., Cagnon C.H., McNitt-Gray M.F. (2003) A Monte Carlo-based method to estimate radiation dose from spiral CT: from phantom testing to patient-specific models, Phys. Med. Biol. 48, 2645-2663.

Kalender W.A. (2014) Dose in X-ray computed tomography, Phys. Med. Biol. 59, 129-150, and references therein.

Karimi Shahri K., Rafat-Motavalli L., Miri-Hakimabad H. (2013) Can the same dose data be estimated from phantoms with different anatomies? Radioprotection 48, 527-544.

Lee C., Williams J., Lee C., Bolch W.E. (2005) The UF series of tomographic computational phantoms of pediatric patients, Med. Phys. 32, 3537-3548.

Khursheed A., Hillier M.C., Shrimpton P.C., Wall B.F. (2002) Influence of patient age on normalized effective doses calculated for CT examinations, Brit. J. Radiol. 75, 819-830.

Lee C. (2006) Development of the voxel computational phantoms of pediatric patients and their application to organ dose assessment, $\mathrm{PhD}$. thesis, University of Florida. 
Lee C., Lee C., Williams J.L., Bolch W.E. (2006) Whole-body voxel phantoms of pediatric patients - UF Series B, Phys. Med. Biol. 51, 4649-4661.

Lee C., Lee C., Staton R.J., Hintenlang D.E., Arreola M.M., Williams J.L., Bolch W.E. (2007) Organ and effective doses in pediatric patients undergoing helical multislice computed tomography examination, Med. Phys. 34, 1858-1873.

Lee C. et al. (2011) Organ doses for reference adult male and female undergoing computed tomography estimated by Monte Carlo simulations, Med. Phys. 38, 1196-1206.

Lee C., Kim K.P., Long D.J., Bolch W.E. (2012) Organ doses for reference pediatric and adolescent patients undergoing computed tomography estimated by Monte Carlo simulation, Med. Phys. 39, 2129-2146.
Staton R.J. et al. (2006) Organ and effective doses in newborn patients during helical multislice computed tomography examination, Phys. Med. Biol. 51, 5151-5166.

USFDA, FDA unveils initiative to reduce unnecessary radiation exposure from medical imaging (2010) http://www.fda.gov/ newsevents/newsroom/pressannouncements/ucm200085.htm.

Zankl M., Veit R., Williams G., Schneider K., Fendel H., Petoussi N., Drexler G. (1988) The construction of computer tomographic phantoms and their application in radiology and radiation protection, Radiat. Env. Biophys. 27, 153-164.

Zankl M., Panzer W., Petoussi N., Drexler G. (1995) Organ doses for children from computed tomographic examinations, Radiat. Prot. Dosim. 57, 393-396.

Cite this article as: P. Akhlaghi, H. Miri-Hakimabad, L. Rafat-Motavalli. Dose estimation in reference and non-reference pediatric patients undergoing computed tomography examinations: a Monte Carlo study. Radioprotection 50(1), 43-54 (2015). 